\title{
FINAL REPORT TO THE DEPARTMENT OF ENERGY
}

Grant \# DE FG 02 87ER45330

June 27,2000

Excitons and plasmas in Semiconducting Microstructures and Ternary Alloys

\author{
M.D. Sturge \\ Dartmouth College \\ Hanover, NH 03755 \\ This report covers the period Sept 15, 1987 to April 30, 2000

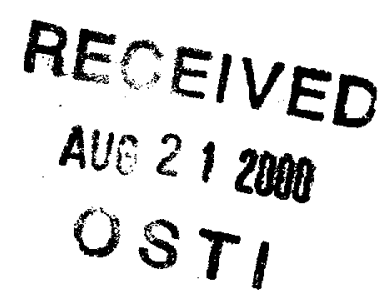

\section{Contents}

Summary

I. Type II short-period AlAs/GaAs superlattices 3

II. Mixed type CdTe/CdZnTe superlattices 5

III. Exciton-phonon coupling at isoelectronic traps 5

IV. Exciton kinetics in strain confined wires and dots 5

V. Exciton-electron interaction in mixed type quantum wells: free 6 trions, magnetically induced transitions, and hidden symmetry.

VI Relaxation processes in quantum wells and at defects $\quad 8$

VII Education $\quad-8$

Acknowledgements $\quad 8$

Publications based on work supported by this grant 9

$\begin{array}{ll}\text { Personnel } & 12\end{array}$

References

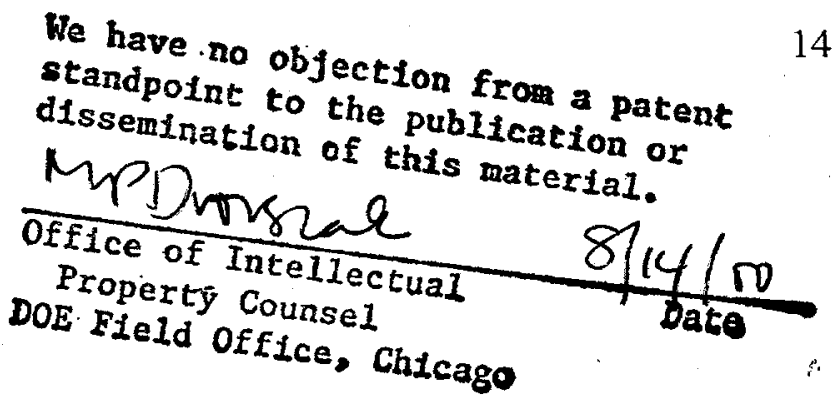




\section{DISCLAIMER}

This report was prepared as an account of work sponsored by an agency of the United States Government. Neither the United States Government nor any agency thereof, nor any of their employees, make any warranty, express or implied, or assumes any legal liability or responsibility for the accuracy, completeness, or usefulness of any information, apparatus, product, or process disclosed, or represents that its use would not infringe privately owned rights. Reference herein to any specific commercial product, process, or service by trade name, trademark, manufacturer, or otherwise does not necessarily constitute or imply its endorsement, recommendation, or favoring by the United States Government or any agency thereof. The views and opinions of authors expressed herein do not necessarily state or reflect those of the United States Government or any agency thereof. 


\section{DISCLAIMER}

Portions of this document may be illegible in electronic image products. Images are produced from the best available original document. 


\section{$\underline{\text { Summary }}$}

This report summarizes the work supported by this grant over a period of more than twelve years, and is necessarily rather brief in its coverage of the early years. For a fuller account of the early work, the annual progress reports submitted to DOE shold be consulted.

The work deals primarily with semiconducting superlattices (SLs), in which two semiconductors with different band gaps are interleaved, with layer thicknesses defined to one monolayer. The electronic and optical properties of the SL are quite different from those of the constituents and offer interesting new possibilities both in device design and in basic physics. This research aims to improve our understanding of optically excited states in SLs. We studied these structures by time-and space-resolved tunable laser spectroscopy, with and without external perturbations such as magnetic field, electric field, and uniaxial stress.

In SLs with only a few atomic layers per period the familiar "effective mass model" of semiconductor states breaks down. We have made precise optical experiments on wellcharacterized material to test current "first principles" calculations of the band structure. Our work under this grant has shown that the material we are using is of sufficiently high quality to test the theoretical predictions. Comparison of theory and experiment provides a new and sensitive probe of the interface quality on a fine scale. Analysis of the temperature dependence of the exciton decay dynamics provides complementary information.

From a careful study of the exciton spectra of mixed type I- type II CdTe/CdZnTe SLs we have obtained the band offset at the $\mathrm{CdTe} / \mathrm{CdZnTe}$ interface to unprecedented accuracy.

A new analysis of exciton-phonon coupling at isoelectronic traps, which provide a threedimensional analog of the exciton traps formed by well width fluctuations in SLs, has corrected errors in the literature and given a self consistent model of phonon-assisted transitions at these centers.

We made a detailed study of exciton kinetics in strain confined systems: i.e. wires and dots created in a quantum well by localized lateral strain. This work has established the mechanism of excitation transfer from the two-dimensional "host" quantum well to the one- or zero-dimensional quantum wire or dot, and identified an intermediate state in which the electron and hole are separated by an energy barrier.

Time- and space-resolved spectroscopic studies of mixed-type multiple quantum wells have led to the definitive identification of a new excitonic complex, the free trion, whose existence had not previously been established unambiguously. We also identified, in these and other samples, a new symmetry-driven transition from "trion like" to "free electron like" behavior of a two-dimensional electron gas in a magnetic field at low temperature, and shown that it can be understood in terms of a "hidden symmetry". 


\section{Type II short-period AlAs/GaAs superlattices}

The lowest exciton in a Type II AlAs/GaAs superlattice (SL) consists of the AlAs X electron and the GaAs $\Gamma$ hole ${ }^{1,2}$, and is indirect in momentum space as well as being spatially separated in real space, so that its decay by photoluminescence (PL) is forbidden to zero'th order by momentum conservation. The transition is made weakly allowed by four possible mechanisms which mix the $\Gamma$ and $X$ electron states: by phonons, by the SL potential, by impurities, and by random scattering at the interface. The first mechanism cannot give a no-phonon line, and since a no-phonon line is in fact observed in PL, at least one of the other three must be present.

In a cubic crystal there are three degenerate $X$ electron states, corresponding to electron momenta directed along the $\mathrm{x}, \mathrm{y}$ or $\mathrm{z}$ directions. In the SL this degeneracy is lifted and we label the resulting states $X_{z}$ and $X_{x y}$, where we have taken the growth direction to be the $\mathrm{z}$ axis. Only $\mathrm{X}_{\mathrm{Z}}$ can be mixed with $\Gamma$ by the SL potential, but any state can be mixed by interface disorder (pubs 88-3,89-1). Electrons relax rapidly and non-radiatively to the lowest X state. The low temperature PL is therefore predominantly from this state, though very weak PL is sometimes observed from the $\Gamma$ state, for which the radiative transition probability is high enough to compete with the non-radiative $\Gamma \rightarrow X$ transfer (pub 91-3).

The questions addressed in the work supported by this grant were:

(1) What is the relative importance of the different mechanisms which make the transition allowed? How large is the mixing of the $\Gamma$ and $X$ states?

(2) (a) What is the order and separation of the $X_{z}$ and $X_{x y}$ states, particularly in very short period SLs where the effective mass approximation might be expected to break down? Recent "first principles" calculations make predictions which need to be tested. In particular, it is theoretically predicted that a state deriving from the $\mathrm{L}$ point of the bulk, rather than the $\mathrm{X}$ point, should be lowest for a $1 / 1 \mathrm{SL}$. Is this the case?

(b) The optical selection rules for excitons depend on the symmetry of the exciton state in the space group of the SL. These both depend on whether the layer thickness (measured in monolayer units) is even or odd. Can these differences be detected?

(c) Can structural information be obtained from PL data?

(3) (a) Are the indirect excitons which are involved in photoluminescence at low temperature localized or delocalized?

(b) If the former, is it possible to detect a mobility edge, as in the case of direct excitons ${ }^{3}$ ?

The work done under this grant has established a firm answer to question (1) and affirmative answers to questions (2a), (b) and (c). For question (3), the exciton dynamics show firm evidence for localization at helium temperature, with delocalization as the temperature is raised; but no evidence for a mobility edge has been found. 


\section{(1) -X mixing:}

In an $\mathrm{X}$-exciton the electron and hole occupy different layers, while in a $\Gamma$-exciton they are both in the GaAs layer. Hence they respond differently to an applied electric field, and the energy levels can be made to cross. If $\Gamma$ and $X$ are mixed the states anticross. This anticrossing has been observed and a mixing matrix element obtained which is consistent with the observed radiative lifetimes of $\mathrm{X}$-excitons (pubs 88-1, 89-1).

(2) Energy levels and symmetry: use of spectroscopy to obtain structural information

Our work under this grant has established the symmetry and energy of the conduction band minimum (CBM) in very short period SL's (period $\leq 8$ monolayers). We find that in our samples the selection rules predicted by the space group symmetry are well obeyed, showing that the samples are indeed true SL's and not just partially ordered alloys, and that the observed transitions are intrinsic and not due to interface defects, as was the case in virtually all previous work in this range of period (pubs 90-1, 91-1, 91$5,92-5,94-1)$. The predictions of the most sophisticated band structure calculations ${ }^{4}$ of the electronic structure are confirmed except in the case of the shortest period ("1/1") superlattices (see the next paragraph). This comparison of theory and experiment constitutes a very searching test, since all the parameters of the theory are determined by data on the constituent bulk semiconductors. [pubs 90-4, 90-5, 91-1, 92-5, 94-1].

For an ideal 1/1 SL (i.e. a SL consisting of successive single monolayers of AlAs and $\mathrm{GaAs}$ ) the band structure calculations predict that the conduction band minimum (CBM) derives from the $\mathrm{L}$ point of bulk GaAs${ }^{4}$. On the other hand, our data show unambiguously that the CBM derives from the $\mathrm{X}$ point (pub. 91-4). Detailed band structure calculations ${ }^{5}$ show that this is to be expected if about $30 \%$ of the $\mathrm{Ga}$ and $\mathrm{Al}$ atoms are interchanged. Since our data show that the selection rules for a perfect superlattice are well obeyed [pubs. 91-1,92-5] the atomic interchanges cannot be random, but are restricted to those which preserve the space group symmetry of the ideal superlattice. Total energy calculations ${ }^{6}$ suggest that this type of interchange is (to a small extent) energetically favored both over the perfect SL and the random alloy. Our results show that PL spectroscopy can provide a useful non-destructive tool for the investigation of interface structure (pub 94-1).

\section{(3) Dynamics of excitons in indirect gap superlattices.}

The no-phonon radiative decay of indirect excitons in a superlattice is made allowed (subject to the selection rules mentioned above) by the SL potential. Because of fluctuations in the interface position, this potential is the sum of terms of random phase, with the result that the matrix element for radiative decay of localized excitons has a Gaussian distribution? This leads to non-exponential decay at low temperatures. As the temperature is raised excitons migrate and the radiative rate is averaged out. We have adapted the dynamical theory of the averaging of chaotic light 8 to this problem and obtained a quantitative fit to the data, from which exciton hopping rates as a function of temperature can be obtained. [pubs 89-1, 93-3]

\section{Mixed type CdTe/CdZnTe quantum wells and superlattices}

This system is of interest for several reasons: it provides a non-magnetic model for the IIVI magnetic semiconductor superlattice, and the light hole $(\mathrm{LH})$ and heavy hole $(\mathrm{HH})$ reside in different layers ${ }^{9}$, so that the $\mathrm{LH}$ exciton is Type II while the HH is Type I. It 
also shows remarkable optical non-linearities at very low excitation ${ }^{10}$. The work was in collaboration with the group of Dr Merle d'Aubigné of the CNRS Laboratory in Grenoble, France.

We analyzed the spectra of compensated strain $\mathrm{CdTe} / \mathrm{Cd} .{ }_{91} \mathrm{Zn}_{.09} \mathrm{Te}$ quantum wells. In this system shear strain, which splits the light hole $(\mathrm{LH})$ from the heavy hole $(\mathrm{HH})$ band and has opposite signs in the CdTe and $\mathrm{Cd}_{.91} \mathrm{Zn}_{.09}$ Te layers, dominates the offset ${ }^{9}$. We showed that the band offset averaged over the $\mathrm{LH}$ and $\mathrm{HH}$ is zero within experimental error, due to a close cancellation between the "chemical" offset (the offset in the absence of strain) and the hydrostatic strain contribution"l (pubs 92-3, 93-2).

From March 1993 the work on this system was supported by NSF and work after that date is not included in this report.

\section{Exciton-phonon coupling at isoelectronic traps}

The exciton bound to an isoelectronic (i.e. electrically neutral) center such as nitrogen in $\mathrm{GaP}$ has much in common with an indirect exciton localized by width fluctuations in a quantum well. Since the bound exciton has an energy which for any given center is well defined rather than depending on random fluctuations, it can provide a useful model for a localized exciton. The publication of a new (and, as it turned out, erroneous) treatment of the phonon sidebands ${ }^{12}$ of these bound excitons stimulated us to re-examine the theory. We have shown that the treatment of ref 12 is internally inconsistent (pub 92-4) and is based on an incorrect analysis of the data (pub 92-1). We have developed a comprehensive theory of these sidebands, based on an improved theory of the isoelectronic center (pub 92-2), which treats coupling to "momentum-conserving" and to "configuration-coordinate" phonons in a consistent manner (pub.93-1) and is in good agreement with experiment.

\section{Exciton kinetics in strain confined wires and dots}

The use of lateral strain confinement as a method of producing low-dimensional structures was pioneered by Dr Kathleen Kash of Bellcore (now at CWRU) in collaboration with the PI (pub 88-2, ref. 13). This technique preserves the high quantum efficiency of the underlying quantum well, and makes possible detailed study of the exciton kinetics and energy transfer processes in a confined system. Initially supported by DOE, this work was supported by ONR from 1991 to 1993, but reverted to DOE upon the expiration of the ONR support.

It has been shown ${ }^{13}$ that strain gradients on a scale of $\sim 100 \mathrm{~nm}$ can produce quantum confinement of excitons to one or zero dimension within semiconductor quantum wells. It has been appreciated for many years that such confinement, to "quantum wires" or "quantum dots", should have important consequences, especially for exciton-exciton and exciton-phonon interactions ${ }^{14}$. Strain confinement avoids many of the difficulties inherent in other confinement techniques, in particular the rapid non-radiative decay associated with etched or ion-bombarded structures. Furthermore it can be used to create systems with unique properties, for example "antenna" systems, where the quantum well can be used as an antenna to funnel excitation to the dots or wire, thus greatly enhancing the optical nonlinearity and the quantum efficiency ${ }^{15}$.

The theory of electron and hole states in an inhomogeneous strain field was put on a firm basis by Zhang ${ }^{15}$ (a student in this group, supported at that time by ONR) His theory predicts a potential barrier to migration of electrons from the unstrained region (the 
"quantum well") to the wire or dot. On the other hand heavy holes experience no such barrier. Our measurements, supported by ONR, of the temperature dependence of the luminescent intensity and decay kinetics of strain-confined quantum wires and dots demonstrated thermally activated energy transfer across a barrier ${ }^{16}$. Our subsequent work, supported by DOE, has shown that the transfer proceeds via an intermediate state in which electrons and holes are spatially separated by the strain potential, the hole having migrated to the dot while the electron is still outside the barrier. This state has an important effect on the non-linear optical behavior of these structures (pubs 94-T1, 95-1, 95-2, 96-1, 96-4, 96-7, 96-T2, 97-2).

The discrete nature of the states in quantum dots has a profound effect on their coupling to phonons. Relaxation between discrete states can only occur through a one-phonon process if a phonon of the required energy has a wavelength comparable to the spatial extent of the state $^{14}$. Such a phonon does not exist in our quantum dots, and as a result we found that at low excitation intensity the thermalization of excitons to their ground state is at least two orders of magnitude slower than in the parent quantum well. This slowing down was not observed in wires of fabricated by the same technique on the same quantum well, showing that it is a consequence of dimensionality, not of the fabrication process. As the excitation is increased, Auger processes 14 become possible when there is more than one electron-hole pair per dot, and the relaxation accelerates. By varying the magnetic field we could tune the inter-level separation and thus study the energy dependence of the relaxation rate. It was found to increase rapidly when the energy separation exceeds 20 $\mathrm{meV}$. This could be due either to the onset of multiphonon relaxation, or to relaxation via nonradiative excitonic states, which become dense in this energy range ${ }^{17}$ (pubs 94-2, 962, 96-T1).

V Exciton-electron interaction in mixed type quantum wells: free trions. magnetically induced transitions, and hidden symmetry

A wide GaAs quantum well with AlAs barriers is "type I", i.e. the lowest electron and hole states are both in the GaAs. However, if the GaAs well is less than about $3 \mathrm{~nm}$ wide, it is "type II", that is, the lowest electron state is in the AlAs, while the hole state remains in the GaAs. In a "mixed type" system a type II well is separated by a moderate thickness $(\sim 10 \mathrm{~nm})$ of AlAs from a wider, type I, GaAs well ${ }^{18}$. If electron-hole pairs are created optically in the narrow well, the electrons can easily transfer to the wide well through the AlAs without encountering any barrier, while the holes have to tunnel through a barrier $\sim 0.5 \mathrm{eV}$ high, so that they transfer slowly ${ }^{18}$. Since the electrons in the wide well cannot recombine until the hole reaches them, a small intensity of light above the narrow well bandgap can create a substantial density of electrons in the wide well. Although some of this weak light is, of course, absorbed in the wide well, the short exciton lifetime $(\sim 1 \mathrm{nsec})$ ensures that the resultant steady state exciton density in the wide well is very small. More intense pulsed excitation at the wide well band gap can then be used to create a controllable density of excitons in the presence of a background electron density.

We found that when electrons are present in the wide well, a new photoluminescence line "Y" is observed, $1.3 \mathrm{meV}$ below the usual heavy hole exciton line. The intensity ratio of these two lines depends on electron density and on temperature, but not on the exciton density. The $Y$ line resembles lines which have been variously attributed to biexcitons ${ }^{19}$, to regions of different layer width ${ }^{20}$, and to excitons bound to localized electrons ${ }^{21,22}$. However, we found that none of these explanations can account for the data. In particular, we found that (1) in equilibrium the two lines have the same decay rate (ruling out biexcitons, whose equilibrium density varies as the square of the exciton density 23 ); 
(2) the radiative rate of the $Y$ line, obtained by measuring the dependnce of the average decay rate on electron density, is a factor of four greater than that of the exciton, and has the same temperature dependence; (3) spatially resolved luminescence measurements showed that above about $5 \mathrm{~K}$ the particle responsible for the $\mathrm{Y}$ line diffuses at about half the rate as the free exciton, and both rates are proportional to temperature, as expected for free classical particles obeying the Einstein relation; (4) unlike the exciton line, the Y line has a Lorentzian profile, showing that the final state is lifetime broadened and hence free rather than bound. All the data can be quantitatively accounted for if the $Y$ line is assigned to the decay of the free negatively charged exciton ("trion"). This entity had previously been tentatively identified in bulk $\mathrm{Si}$ and $\mathrm{Ge}^{24}$, but putative identifications in $\mathrm{GaAs}^{25}$ were questionable. To our knowledge our data provided the first unambiguous evidence for free (as opposed to bound) trions in a 2-D system. (pubs 96-5, 96-6, 96-8, 97-1, 00-3).

At higher electron density excitons and trions can no longer form, because of screening and band filling 26 . At a certain density (about $3 \times 10^{10} \mathrm{~cm}^{-2}$ in our case) a "Mott transition" occurs, and above this density excitons and trions no longer exist: instead a free hole recombines with a Fermi gas of electrons. The emission spectrum then has a width determined by the Fermi energy. We found that this transition is rather gradual and has no marked effect on the temporal or spatial kinetics: the lifetime and diffusivity of the hole were found to be indistinguishable from those of the trion.

A magnetic field shrinks the exciton and trion wave functions, reducing the effect of screening and band filling, and thus might be expected to increase the density at which the Mott transition occurs. This expectation is apparently fulfilled: at a certain field the spectrum of broad transitions between Landau levels (LLs), characteristic of a free holes in an electron gas, abruptly changes to a spectrum almost indistinguishable from the trion spectrum observed in the same field at low electron density. Such a transition from "LLlike" to "trion-like" behavior in doped SLs has been reported by other authors ${ }^{27}$. Other changes occur at the same field: the emission line narrows, and various satellite lines, which are strong at lower field, disappear or become very weak ${ }^{28}$. However, when we compared data at different electron densities, we found a remarkable correlation: the field at which the transition occurs is that at which the lowest LL for electrons is exactly filled; in the conventional parlance, the "filling factor" $v=2$ at the transition. At larger $v$, (i.e at lower field or higher density) the spectrum is LL-like: at lower $v$, it is trion-like. In the model mentioned above, where the transition is due to the magnetically induced change in screening and band-filling, there is no reason to expect such a correlation, yet it holds over too wide a range in electron density (a factor of ten) to be coincidental (pub 97-3).

The solution to this enigma has been found by looking at the problem from the high field point of view instead of the low field. A two-dimensional system at very high magnetic field, in which the highest occupied hole and electron LLs have the same quantum number $\mathrm{n}$, has been shown to possess a hidden symmetry ${ }^{29}$ (somewhat analogous to the hidden symmetry responsible for the angular momentum degeneracy of hydrogenic states). Because of this symmetry the only possible optical transition is one in which an electron-hole pair ("magneto-exciton") is created or destroyed: furthermore the energy of this transition is independent of the number of electrons and holes present,there being an exact cancellation of the various terms in the electron-electron and electron-hole interaction. Although this symmetry is only exact at infinite field, it has been found to account for the spectra of highly excited quantum wells at quite moderate fields ${ }^{30}$. If we assume that hidden symmetry provides a correct classification of the states of an electron gas at the moderate magnetic fields considered here, we can understand why the 
transition always occurs at $v=2$. When $v<2$, electrons and holes both occupy their lowest $(n=0) L L$, so that hidden symmetry holds, at least approximately. When $v>2$, on the other hand, electrons occupy the $n=1 \mathrm{LL}$, while the small number of photexcited holes still have $n=0$, so that the hidden symmetry is broken. There is now no requirement that the optical transition coincide with that at low electron density, and a host of new transitions, involving the creation of magnetoplasmons, shake up of electrons to higher LLs ${ }^{28}$, scattering of magnetoexcitons by $n=1$ electrons, and creation of magneto-excitons with $\mathrm{k} \neq 0$ by scattering from electrons, become allowed. Calculations show that these transitions can be quite strong, and the observed dependence of their intensity on field 28 can be accounted for by a model based on broken hidden symmetry (pubs 00-1, 00-2, 00-3).

VI Relaxation processes in quantum wells and at defects.

In his first year at Dartmouth, Dr Ge completed the theoretical work on hot carriers and non-radiative recombination which he had begun while in China (pubs 90-2, 90-3, 90$6)$.

\section{Education}

An important part of this work has been the introduction of undergraduates to experimental research in the laboratory. During the budget period 25 undergraduate interns (listed at the end of this report) have worked in the lab, supported in part by the grant. Several have done independent study projects and two have written senior theses. The majority of these students continued on to graduate school.

\section{Acknowledgements}

This work would not have been possible without the generous support of several crystal growers besides Dr Tamargo: in particular Drs J.P. Harbison, B. P. van der Gaag, A. S. Gozdz and Ms L. T. Florez, Bellcore; Dr L.N. Pfeiffer and Mr K. West, Bell Labs; Dr C.T. Foxon, Philips Research Laboratories, England; and Drs. H. Mariette and N. Magnea, CNRS and CEN, Grenoble, France. I am grateful to the following colleagues for many valuable discussions: Professor L.J.Sham, UCSD; Professors W.E.Lawrence, C.G.Levey, G. Nunes and J.E.Walsh, Dartmouth; Drs A. Zunger, NREL; P. Hawrylak, NRC Ottawa; Y. Merle d'Aubigné, R. Romestain, G. Fishman, P.Peyla, R.T. Cox and Le Si Dang, Grenoble. My postdocs Drs Colette Maloney and A.Naumov gave essential guidance to generations of graduate students and made important contributions to the running of the laboratory. In the early stages of this work my then co-PIs Drs R.E.Nahory and Maria C. Tamargo, and also Drs J.M. Worlock, MarieHélène Meynadier and Kathleen Kash, all then at Bellcore, made invaluable contributions. 


\section{Publications}

The following publications are based wholly or in part on work supported by this grant.

88-1 Indirect-direct anticrossing in GaAs/AlAs superlattices induced by quantum confined Stark effect: evidence of $\Gamma$-X mixing. Marie-Hélène Meynadier, R E Nahory, J.M.Worlock, Maria C Tamargo, J.L.de Miguel and M D Sturge, Phys. Rev. Lett. 60, 1338 (1988).

88-2 Strain-induced lateral confinement of excitons in GaAs/AlGaAs quantum well microstructures. Kathleen Kash, J.M.Worlock, M.D.Sturge, P.Grabbe, J.P.Harbison, A.Scherer, and P.S.Lin, Appl. Phys. Lett. 53, 782 (1988).

88-3 Indirect excitons in short period superlattices. M.D.Sturge, E.Finkman and M.C.Tamargo, J.Lumin. 40/41, 425 (1988).

89-1 Photoluminescence decay time studies of type II GaAs/AlAs quantum well structures. M.D.Sturge, Janet L. Mackay, Colette Maloney and J.K.Pribram. J. Appl. Phys. 66, 5639 (1989)

89-2 Introduction to the optical properties of quantum wells and superlattices. M.D.Sturge and MH Meynadier. J. Lumin. 44, 199 (1989).

90-1 Optical transitions in very short period superlattices. Weikun Ge, W.D.Schmidt, Yong Zhang, Janet L. Mackay and M.D.Sturge, in Lattice Dynamics and Semiconductor Physics (Festschrift for Professor Kun Huang, ed. J.Xia et al.) p.383 (1990).

90-2 Hot carrier relaxation processes and nonequilibrium phonon effect in multiple quantum well structures. Weikun Ge, Z Xu, Y Li, Z Xu, J Xu, B Zheng and W Zhuang, J. Lumin. 46, 137 (1990).

90-3 High pressure behavior of electronic states in $\mathrm{GaAs} / \mathrm{Ga}_{1-\mathrm{x}} \mathrm{Al}_{\mathrm{x}} \mathrm{As}$ multiple quantum wells. L. Wang, R. Tang, J. Hu, Y. Wang, W. Jia, Weikun Ge and B. Wang. Superlattices and Microstructures 7, 175 (1990).

90-4 Energy levels of very short period (GaAs) $)_{n}(\mathrm{AlAs})_{\mathrm{n}}$ superlattices. Weikun Ge, M.D.Sturge,W.D.Schmidt, L.N.Pfeiffer and K.W.West. Appl. Phys. Lett. 57, 55 (1990).

90-5 Energy levels of very short period GaAs-AlAs superlattices, M.D.Sturge, Weikun Ge, W.D.Schmidt, L. N. Pfeiffer and K.W. West, Proc 20th ICPS, ed. E.M.Anastassakis and J.D.Joannopoulos (World Scientific, Singapore, 1990). p. 1029.

90-6 EL2 ionization induced interconversion among three Ga-O-Ga LVM absorption bands in semi-insulating GaAs.

Weikun Ge and Chung-ying Song. Proc. 6th Conf Semi-Insulating III-V Compounds, Toronto, p. 41 (1990).

91-1 Symmetry of the lowest conduction band states in very short period GaAs-AlAs superlattices. Weikun Ge, W.D.Schmidt, M.D.Sturge, L. N. Pfeiffer and K.W. West, J. Lumin. 48/49,759(1991).

91-2 Type II indirect gap superlattices under intense optical excitation. Janet. L. Mackay, M. D. Sturge, M.- H. Meynadier, M. C. Tamargo, and J.L. de Miguel, Proc. ICL90, J. Lumin. 48/49,731 (1991).

91-3. $\Gamma \rightarrow \Gamma$ emission from indirect gap very short period GaAs/AlAs superlattices. Weikun Ge, Janet. L. Mackay,L. N.Pfeiffer and K. W. West, J. Lumin. 50, 133 (1991).

91-4 Conduction band minimum of $(\mathrm{GaAs})_{1}-(\mathrm{AlAs})_{1}$ superlattices: relation to $\mathrm{X}$. Weikun Ge, W.D.Schmidt, M.D.Sturge, L. N. Pfeiffer and K. W. West, Phys. Rev. B44, 3432 (1991).

92-1 Comment on "Radiative and nonradiative recombination of bound excitons in GaP:N, I. Temperature behavior of zero-phonon line and phonon sidebands of bound excitons", M.D.Sturge and E. Cohen, Phys. Rev. B45, 11370 (1992). 
92-2 Acceptor-like bound exciton in semiconductors. Yong Zhang, Phys. Rev. B45, 6809 (1992).

92-3 Exciton binding energies and the valence band offset in mixed Type I- Type II strained layer superlattices, P. Peyla, Y. Merle d'Aubigné, A. Wasiela, R. Romestain, H. Mariette, N. Magnea, H. Tuffigo, and M.D.Sturge, Phys. Rev.B 46,1557 (1992).

92-4 Comment on "Radiative and nonradiative recombination of bound excitons in GaP: $\mathrm{N}$ ". Weikun Ge, Yong Zhang, Donglin Mi, Jainsheng Zheng, Bingzhang Yan, and Boxi Wu, Phys. Rev. B46, 5004, 1992.

92-5 Space group symmetry and parity of the conduction band minimum in very short-period. GaAs-AlAs superlattices, Weikun Ge, W.D.Schmidt, M.D.Sturge, L. N.Pfeiffer and K. W. West, Solid State Commun. 82, 951 (1992).

93-1 On the phonon sidebands of excitons bound to isoelectronic impurities in semiconductors, Yong Zhang, Weikun Ge, M. D. Sturge, Jiansheng Zheng, and Boxi Wu, Phys.

Rev.B47, 6330 (1993).

93-2 Excitonic effects in mixed Type I - Type II CdTe-CdZnTe superlattices, P. Peyla, V. Chitta, A. Wasiela, Y. Merle d'Aubigné, H. Mariette, J.M.Berroir, M.D.Sturge, and N. Magnea. To appear in Physics of semiconductors (Proc. 21st $I C P S$ ) ed. B. Sapoval (Springer Verlag, 1993).

93-3 Temperature dependent radiative decay of localized excitons in a type II GaAs/AlAs superlattice, J.F.Angell and M.D.Sturge. Phys. Rev.B 48, 4650 (1993).

94-1 Electronic states in GaAs-AlAs short-period superlattices: energy levels and symmetry, Weikun Ge, W.D.Schmidt, M.D.Sturge, L. N. Pfeiffer and K. W. West, J. Luminescence 59, 163 (1994).

94-2 The diamagnetic shift of excitons in strain-confined quantum wires and dots. T. H. Gfroerer, M. D. Sturge, K. Kash, J. A. Yater, A. S. Plaut, P. S. D. Lin, L. T. Florez, J. P. Harbison, S. R. Das, and L. Lebrun. Proc. 11th International Conf.on Applications of High Magnetic Fields in Semiconductor Physics. ed. M. Heiman (Cambridge MA, 1994), p.488..

95-1 Strain-Confined Wires and Dots at a GaAs/Al $\mathrm{XGa}_{1-\mathrm{X}}$ As Interface.

Yitong Gu, Yong Zhang, M.D.Sturge, K. Kash, B.P. Van der Gaag, A.S. Gozdz, L.T.Florez and J.P.Harbison. Superlattices and Microstructures 17, 67 (1995).

95-2 Temperature Dependent Time-resolved Exciton Luminescence in GaAs/Al $\mathrm{X} \mathrm{Ga}$ 1$\mathrm{x}$ As Quantum Wires and Dots. Yong Zhang, M. D. Sturge, K. Kash, B. P. van der Gaag, A. S. Gozdz, L. T. Florez and J. P. Harbison. Superlattices and Microstructures 17, 201 (1995).

96-1. Long-lived intermediate state in strain-confined quantum dots. Yitong Gu, M. D. Sturge, K. Kash, B. P. van der Gaag, A. S. Gozdz, L. T. Florez and J. P. Harbison. Superlattices and Microstructures, 19, 131 (1996)

96-2 Slow relaxation of excited states in strain-induced quantum dots, T. H. Gfroerer, M. D. Sturge, K. Kash, J. A. Yater, A. S. Plaut, P. S. D. Lin, L. T. Florez, J. P. Harbison, S. R. Das, and L. Lebrun. Phys. Rev B53, 16474 (1996).

96-3 Thermodynamics and diffusion of free trions in mixed type GaAs/AlAs quantum wells, H. W. Yoon, Arza Ron, M. D. Sturge, L.N. Pfeiffer. Proceedings of the MRS Symposium on Optoelectronic Materials, vol. 417, 309 (1996).

96-4 Optical studies of carrier transfer in a strain-induced quantum dot. Yitong Gu, M.D. Sturge, K. Kash, B.P. Van der Gaag, A.S. Gozdz, L.T.Florez and J.P.Harbison. Proceedings of the MRS Symposium on Optoelectronic Materials, vol. 417, 159 (1996).

96-5 Thermodynamics of trions in mixed type GaAs/AlAs quantum wells. Arza Ron, H. Yoon, M.D.Sturge, A. Manassen, E. Cohen and L.N.Pfeiffer, Solid State Commun.97 741 (1996).

96-6. Diffusion of free trions in mixed type GaAs/AlAs quantum wells. H. W. Yoon, Arza Ron, M. D. Sturge, L.N. Pfeiffer. Solid State Commun. 100, 743 (1996). 
96-7 Optical study of carrier transfer in strain-induced quantum dots Yitong Gu; Sturge, M.D.; Kash, K.; Van der Gaag, B.P.; Gozdz, A.S.; Florez, L.T.; Harbison, J.P.Symposium on Optoelectronic Materials: Ordering, Composition Modulation, and Self-Assembled Structures. p.159 Eds: Jones, E.D.; Mascarenhas, A.; Petroff, P. (1996)

96-8 Thermodynamics and diffusion of free trions in mixed type GaAs/AlAs quantum wells, Yoon, H.W.; Ron, A.; Sturge, M.D.; Pfeiffer, L.N. Symposium on Optoelectronic Materials: Ordering, Composition Modulation, and Self-Assembled Structures, eds: Jones, E.D.; Mascarenhas, A.; Petroff, P. (1996)p.309.

97-1 Diffusion of trions in mixed type GaAs/AlAs quantum wells, H. W. Yoon, M. D. Sturge, Arza Ron, L.N. Pfeiffer. J. Lumin.72-74, 302 (1997).

97-2 Charge-separated state in strain-induced quantum dots. Yitong Gu, M.D. Sturge, K. Kash, B.P. Van der Gaag, A.S.Gozdz, L.T.Florez and J.P.Harbison. Appl. Phys. Lett.70, 1733 (1997).

97-3 Magnetically induced Mott transition in mixed-type GaAs/AlAs quantum wells. H.W. Yoon, M.D.Sturge and L.N.Pfeiffer. Solid State Commun. 104, 287 (1997).

00-1 Hidden symmetry and the magnetically induced "Mott transition" in GaAs/AlGaAs quantum wells containing an electron gas. E.I Rashba, M.D.Sturge, H.W.Yoon and L.N.Pfeiffer. Solid State Commun.114, 593 (2000)

00-2 Hidden symmetry and magnetospectroscopy of quantum wells near filling factor $n=2$. E.I. Rashba and M.D.Sturge, submitted Phys. Rev B (2000) [at present being revised in response ot referees' reports]

00-3 Recombination emission from 2D trions. E.I. Rashba and M. D. Sturge, in preparation.

Theses

90-T1 A digital data handling system for the Cary spectrophotometer, J.Pringle, Senior Thesis, June 1990.

91-T1 A photo-excited three component electron-hole plasma in type II GaAs/AlAs quantum wells.

Janet L. Mackay, Ph D Thesis, 1991.

92-T1 Localization of excitons in an indirect gap GaAs/AlAs superlattice, J.F.Angell, Ph D Thesis, Sept 1992.

93-T1 Development of a fast pulse diode laser. Irene Georgakoudi, Senior Thesis, June 1993.

94-T1 Strain-confined GaAs/AlGaAs quantum wires and dots. Yong Zhang, $\mathrm{Ph} \mathrm{D}$ Thesis, 1994

96-T1 Magneto-spectroscopy of strain-induced quantum wires and dots. Timothy Gfroerer, Ph D Thesis, 1996.

96-T2 Excitation transfer in strain-induced quantum wires and dots. Yitong Gu, Ph D Thesis, 1996. 


\section{Personnel}

Principal Investigator(s)

Professor Emeritus M.D.Sturge (PhD Cambridge, England, 1957) spent approximately $25 \%$ of his time on this research throughout the period of this grant. The cost of this was borne entirely by the grantee.

Dr R.E.Nahory was Manager and Dr Maria C. Tamargo was a member of the Semiconductor Materials Research Group of Bellcore. They not only provided much of the material used in the early stages of this project but also provided an essential link to related work at Bellcore. Because of Bellcore's change in direction they withdrew from participation in this work in 1991.

Research associate professor Dr Weikun Ge (PhD Manchester, England, 1983) came to Dartmouth from the Institute of Semiconductors in Beijing, where he was an Associate Professor and Associate Director. He was employed on this grant full time from Dec 1988 till Dec 1992, and part time from Jan to Sept 1993. His experimental work was on the energy levels of very short period superlattices; he also did theoretical work on the EL2 defect in GaAs, on exciton-phonon interactions at defects, and on hot carrier effects in quantum wells. He is now an Associate Professor at the University of Science and Technology in Hong Kong.

\section{Visiting research professors}

Professor Arza Ron (PhD Technion, Israel, 1961), was a member of the group for three months in 1995 while on sabbatical leave from the Technion, Haifa, Israel, where she is Professor of Chemistry. While at Dartmouth Professor Ron collaborated with Dr Yoon on the work on mixed type quantum wells mentioned in section III. Only her research expenses were provided from the grant.

Professor Emmanuel I. Rashba (DSc Leningrad, 1963), was a co-founder of the Landau Institute for Theoretical Physics in Chernogolovka, where he was Professor of theoretical and mathematical physics and head of the Semiconductor Theory Division from 1966 to 1992. He then joined the Physics Department of the University of Utah as a research professor, but was forced by illness to retire in 1997 . He was a part-time visiting research professor in the Physics Department at Dartmouth from October 1999 to February 2000, supported by DOE, and is now an adjunct professor in this department. Professor Rashba has been awarded numerous prizes and honors, the most recent being the prize of the International Conference on Luminescence, Tokyo 1999.

\section{Visiting research associate professors}

Professor John K. Pribram (PhD U Mass, 1973) spent the academic year 1987-88 in the group, while on sabbatical leave from Bates College. Part of his research expenses were provided from the grant.

Professor Peter L. Scott (PhD Berkeley, 1963) spent the winter term of 1990 in the group, while on sabbatical leave from UCSC. Part of his research expenses were provided by the grant.

\section{Post-doctoral Research Associate}

Dr Howard Yoon obtained his BA in Physics and Chemistry from Swarthmore College in 1985 and was a Senior Staff Technologist at Bellcore 1985-7. He obtained his PhD in Physics from the University of Illinois in 1994. He was supported by DOE from May 1994 until February 1997. He is now a project leader in the Optical Technology Division at NIST. 
Graduate students

Janet L. Mackay, MS 1987 (Yale U.) was a member of the group from 1988, supported by an AAUW fellowship until June 1991 and then by DOE until October 1991, when she obtained her Ph.D. All her research expenses were provided by the grant. She is now employed by IBM in Burlington VT.

W.David Schmidt, MS 1986 (Indiana U. of PA), was supported by this grant from Oct 1987 till Sept 1991, when he left to take up employment with American Optical. He was primarily responsible for building the laboratory in which most of the work reported here was done, and played an essential role in the experiments on Type II superlattices, but never completed his thesis.

James Angell, BS 1986 (E.Nazarene College), was a member of the group from 1988, supported first by Dartmouth College and then by this grant from July 1991 to Sept 1992, when he obtained his Ph.D. All his research expenses were provided by the grant. He is now employed by Resonetics, an industrial laser company in Nashua NH.

Donglin Mi, MS 1989 (Xiamen U., PRC) was supported by DOE from July 1991 to Dec 1992, when he transferred to NSF support. He was awarded an MS in 1995 and left to work for a software company.

Yong Zhang, MS 1985 (Xiamen U., PRC), was supported by this grant after the expiration of his ONR support in 1993. Originally an exchange student, he transferred to Dartmouth in 1989. He completed his thesis on strain confined excitons and obtained his $\mathrm{PhD}$ in 1994. He is now on the staff of NREL.

Timothy Gfroerer, BS 1989 (University of the South), MSEE 1991 (Georgia Tech) was supported by this grant from 1992 until January 1996, when he obtained his PhD and joined the group of Dr Eric Cornell at JILA as a postdoctoral fellow. He is now an assistant professor at Davidson College.

Yitong Gu, BS 1989 (Fudan U., PRC), was supported by this grant from 1993 until he obtained his PhD in May 1996. He is at present employed in the laser industry.

Undergraduate interns supported in part by this grant

Anthony Cho '89

Kenji Cunnion '89

Jeffrey Buchsbaum '89

Sing Foong Cheah '90 (graduated in 1989)

James Pringle '90

Dan Kaplan '90

Monica Davis ' 90

Jeff Hanissian '90 Robert Parrott '96

Alicia Wilson ' 91

Kirsten Lorentzen '91

Deborah Koh '92

Kenneth Bilchick '93 Theodore Yuo '98 Irene Georgakoudi ' 93
Gerard Vernes '94

Radu Bacioiu '94

Daniel Schwartz '94

Zahir Daya '95

Kimberly Schmitt '95

Joy. Ko '96

Simone Ferdinand '96

David Robinson '96

Cailin Nelson '97

Lily Liu '97 


\section{References}

1 P.Dawson, B.A.Wilson, C.W.Tu, R.C.Miller, Appl. Phys. Lett. 48, 541 (1986); B.A. Wilson, IEEE J. Quant. Electron. 24, 1763 (1988).

2 E.Finkman, M.D.Sturge, M.C.Tamargo, Appl.Phys. Lett. 49, 1299 (1986); E. Finkman, M.D.Sturge, M.-H. Meynadier, R.E. Nahory, M.C.Tamargo, D.M.Hwang, C.C.Chang, J. Lumin. 39, 57 (1987).

3 J.Hegarty, M.D.Sturge. JOSA B2, 1143 (1985)

${ }^{4}$ S-H Wei, A. Zunger, J. Appl. Phys. 63, 5794 (1988):S.B.Zhang, M.S.Hybertson, M.L.Cohen, S.G.Louie, D.Tomanek, Phys. Rev. Lett. B63, 1495 (1989); G.P.Srivastava, R. J. Gordon, Z. Ikonic, Superlatt. Microstr. 9,43 (1991).

${ }^{5}$ L. J. Sham, Yan-Ten Lu, J.Lumin. 44, 207 (1989); A. Zunger, D. B. Laks, Phys. Rev. B45, 11411 (1992)

6 D. B. Laks, A. Zunger Phys. Rev, B45, 14177 (1992)

7 M.V.Klein, M.D.Sturge, E. Cohen, Phys. Rev B25, 4331 (1982); M.D.Sturge, E. Cohen, R.A.Logan, Phys. Rev. B27, 2362 (1983); F.Minami, K. Hirata, K.Era, T.Yao, Y. Masumoto, Phys. Rev. B35, 2875 (1987).

8 M. Jakeman, E.R.Pike, J. Phys. A1, 128 (1968).

9 Y. Merle d'Aubigné, H.Mariette, H.Tuffigo, R.T.Cox, G.Lentz, Le Si Dang, J.L.Pautrat, A.Waliesa, J. Cryst. Growth 101, 650 (1990).

10 A. Naumov, Donglin Mi, M. D. Sturge, Weikun Ge, Le Si Dang, H. Mariette, N.Magnea, J.Appl. Phys. 78, 1196 (1995)

11J. Tersoff, Phys. Rev. Lett. 56, 2755 (1986); C.G.van de Walle, Phys. Rev. 39, 1871 (1989).

${ }^{12}$ X.Zhang, K Dou, Q Hong, M Balkanski, Phys. Rev. B41, 1376 (1990);Q.Hong, X.Zhang, K Dou, Phys. Rev. B41, 2931 (1990); see also H.Chang (X.Zhang), C. Hirlimann, M. Kanehisa, M. Balkanski, Scientia Sinica (ser.A) 25, 942 (1982).

${ }^{13}$ K.Kash, J. Lumin. 46, 69 (1990); K.Kash, B.P.van der Gaag, D.D.Mahoney, A.S.Godz, L.T.Florez, J.P. Harbison, M.D.Sturge, Phys. Rev. Lett. 67, 1326 (1991).

${ }^{14}$ U. Bockelmann, Phys. Rev. B48, 17637 (1993);U. Bockelmann, T. Egeler, Phys. Rev. B46, 15574 (1992)

${ }^{15}$ Yong Zhang, Phys. Rev. B49, 14352 (1994)

16 Yong Zhang, M.D.Sturge, K.Kash, B.P.van der Gaag, A.S.Gozdz, L.T.Florez, J.P. Harbison, J. Lumin. 60/61, 400 (1994); Phys.Rev. B51, 13303 (1995); Superlatt. Microstr. 17, 201 (1995)

${ }^{17}$ U. Bockelmann, Phys. Rev. B50, 1727.1 (1994)

18I. Galbraith, P. Dawson, C.T.Foxon, Phys Rev B45, 13499 (1992); E.Cohen, Arza Ron, E.Linder, L.N.Pfeiffer, Superlatt. Microstruct. 15, 175 (1994); Y. Garini, M.Katz, E. Cohen, E. Ehrenfreund, D. Gershoni, Arza Ron, L.N.Pfeiffer, Proc. 22nd International Conference on the Physics of Semiconductors, ed D.J.Lockwood (World Scientific 1995) p. 1153.

${ }^{19}$ R.C.Miller, D.A. Kleinman, A.C.Gossard, O. Munteanu, Phys Rev B25, 6545 (1982)

${ }^{20}$ M. Koch, G. Bastian, R. Hellmann, J. Feldmann, E.O. Goebel, P. Dawson, Phys. Stat. Sol. B188, 485 (1995); Phys. Rev. B51, 13887 (1995)

${ }^{21}$ G. Finkelstein, H. Shtrikman, I. Bar-Joseph, Phys. Rev. Lett. 74, 976 (1995)

${ }^{22}$ K.Kheng, R.T.Cox, Y. Merle d'Aubigné, F. Bassani, K. Saminadayar, S. Tatarenko, Phys. Rev. Lett. 71, 1752 (1993)

23J.C.Kim. D.R.Wake, J.P.Wolfe, Phys. Rev. B50, 15099 (1994)

${ }^{24}$ V. Timofeev, in Excitons, ed. E.I.Rashba, M.D.Sturge (North Holland Press, Amsterdam, 1982) p. 349

${ }^{25}$ H.Buhmann, L.Mansouri, J.Wang, P.H.Beton, N.Mori, L.Eaves, M.Henini, M. Potemski, Phys. Rev. B51, 7969 (1995)

26. R.C.Miller, D.A.Kleinman, J. Lumin. 30, 520 (1985)

${ }^{27}$ C. H. Perry, J. M. Worlock, M. C. Smith, A. Petrou, in High Magnetic Fields in Semiconductor Physics, edited by G. Landwehr (Springer, Berlin, 1987), p. 202; D. Gekhtman, E. Cohen, Arza Ron, L.N. Pfeiffer, Phys. Rev. B54, R1 (1996); S.R.Ryu, W.-Y.Yu, L.P.Fu, Z.X.Jiang, A.Petrou, B.D.McCombe, W. Schaff, Surf. Sci. 361, 363 (1996). 
${ }^{28}$ M. S. Skolnick, K. J. Nash, M. K. Saker, S. J. Bass, Phys. Rev. B 50, 11771 (1994); G. Finkelstein, H. Shtrikman, I. Bar-Joseph, Phys. Rev. B 56, 10326 (1997).

${ }^{29}$ A. B. Dzyubenko, Yu. E. Lozovik, J. Phys. A: Math. Gen., 24, 414 (1991); V. M. Apal'kov, E. I. Rashba, JETP Lett. 53, 155 (1991); A. H. MacDonald, E. H. Rezayi, D. Keller, Phys. Rev. Lett. 68, 1939 (1992)

${ }^{30}$ L. V. Butov, V. D. Kulakovskii, E. I. Rashba, JETP Letters 53, 109 (1991); L. V. Butov, V. D. Kulakovskii, JETP Lett., 53, 466 (1991); E. I. Rashba, J. L. Birman, Solid State Commun., 84, 99 (1992) 